\title{
Segmental Pulmonary Vein Antrum Isolation Using the "Large-Size" Lasso Catheter in Patients With Atrial Fibrillation
}

\author{
Teiichi Yamane, MD; Taro Date, MD; Yasuko Kanzaki, MD; Keiichi Inada, MD; \\ Seiichiro Matsuo, MD; Kenri Shibayama, MD; Satoru Miyanaga, MD; \\ Hidekazu Miyazaki, MD; Ken-ichi Sugimoto, MD; Seibu Mochizuki, MD
}

\begin{abstract}
Background The limited efficacy and complications of segmental ostial pulmonary vein isolation (PVI) for treating atrial fibrillation (AF) have been discussed so, in the present study the feasibility and efficiency of performing segmental pulmonary vein (PV) antrum isolation to treat $\mathrm{AF}$ were assessed.

Methods and Results A total of 187 patients with drug-refractory AF (paroxysmal 120, persistent 67) underwent segmental PVI guided by circumferential 20-electrode catheters (Lasso). Radiofrequency (RF) current was delivered either at the ostium using a regular Lasso $(15-20 \mathrm{~mm}$ in diameter, 70 patients: Group 1) or at the antrum using a larger Lasso $(25-30 \mathrm{~mm}$ in diameter, 117 patients: Group 2). A significantly wider region had to be ablated, with a longer RF application time, to isolate all 4 PVs in Group 2 patients than in Group 1 patients. Although the rate of recurrence of $\mathrm{AF}$ after the initial session was equal in both groups, a significantly greater number of patients were free from AF after a mean of 1.4 procedures in Group 2 than in Group $1(93 \%$ vs $76 \%$ for paroxysmal $\mathrm{AF}, 78 \%$ vs $48 \%$ for persistent $\mathrm{AF}$ ).

Conclusions Segmental antral PVI using large-sized Lasso catheters was found to be more effective and safer than ostial PVI for the treatment of AF. (Circ J 2007; 71: 753-760)
\end{abstract}

Key Words: Antrum isolation; Atrial fibrillation; Catheter ablation; Pulmonary veins

$\Delta$ lthough the efficiency of ostial pulmonary vein isolation (OPVI) to suppress the occurrence of atrial fibrillation (AF) is well established, the relatively frequent recurrence of $\mathrm{AF}^{2}$ and the appearance of pulmonary vein $(\mathrm{PV})$ stenosis $^{3}$ have recently been highlighted as major limitations of this approach. Because the isolation of the PVs, together with their surrounding tissue, might overcome these problems, at least in part, several approaches using linear ablation techniques, such as circumferential linear ablation using electroanatomical mapping 4 or circumferential $2 \times 2 \mathrm{PV}$ isolation (PVI), have so far been developed; however, there have only been a few reports describing the effects of segmental radiofrequency (RF) application outside the PV ostium6 In this report, we describe the feasibility and efficiency of performing segmental PV antrum isolation (PVAI) using large-sized Lasso catheters compared with the conventional OPVI method.

\section{Methods}

\section{Patient Population}

This study included 187 consecutive patients who underwent PV mapping and ablation for multidrug-resistant AF (paroxysmal AF: 120 patients; persistent AF: 67 patients) and thereafter were observed for at least 12 months

(Received October 20, 2006; revised manuscript received January 24, 2007; accepted February 22, 2007)

Department of Cardiology, Jikei University School of Medicine, Tokyo, Japan

Mailing address: Teiichi Yamane, MD, Department of Cardiology, Jikei University School of Medicine, 3-25-8 Nishi-shinbashi, Minato-

ku, Tokyo 105-8461, Japan. E-mail: yamanet1@aol.com
(Table 1). We defined persistent $\mathrm{AF}$ as an episode of $\mathrm{AF}$ that lasted for more than 14 days and required cardioversion to restore sinus rhythm (SR). Cases of long-lasting persistent AF for more than 12 months were not included. The study group comprised 144 males and 43 females with a mean age of $55 \pm 8$ years. Forty-five patients had evidence of cardiovascular diseases: 32 had hypertension, 9 had coronary artery disease, 5 had dilated cardiomyopathy, and 3 had mitral valve regurgitation. Two types of mapping and ablation techniques were used for PVI. In Group 1, which comprised the first 70 consecutive patients (52 males, mean age: $52.0 \pm 10.3$ years), each PV was disconnected from the left atrium (LA) at its ostium. In Group 2, which included the subsequent 117 patients (92 males, mean age: 53.2 \pm 9.8 years), PVI was performed at its antrum, targeting not only the PV itself but also the surrounding tissues. All patients underwent the ablation procedure and subsequent observa-

Table 1 Comparison of Patient Characteristics in the 2 Groups

\begin{tabular}{lccc}
\hline \hline & $\begin{array}{c}\text { Group 1 } \\
(n=70)\end{array}$ & $\begin{array}{c}\text { Group 2 } \\
(n=117)\end{array}$ & p value \\
\hline Age (years) & $52.0 \pm 10.3$ & $53.2 \pm 9.8$ & $N S$ \\
Sex $(M / F)$ & $52 / 18$ & $92 \pm 25$ & $N S$ \\
Paroxysmal/persistent AF & $44 / 26$ & $79 / 38$ & $N S$ \\
Organic heart disease & 16 & 29 & $N S$ \\
LA diameter (mm) & $38.5 \pm 4.2$ & $39.5 \pm 6.0$ & $N S$ \\
Follow-up (days) & $1,015 \pm 257$ & $647 \pm 197$ & $<0.01$ \\
\hline
\end{tabular}

Group 1 represents patients who underwent ostial pulmonary vein isolation; Group 2 represents patients who underwent pulmonary vein antrum isolation.

AF, atrial fibrillation; LA, left atrial. 
(A)
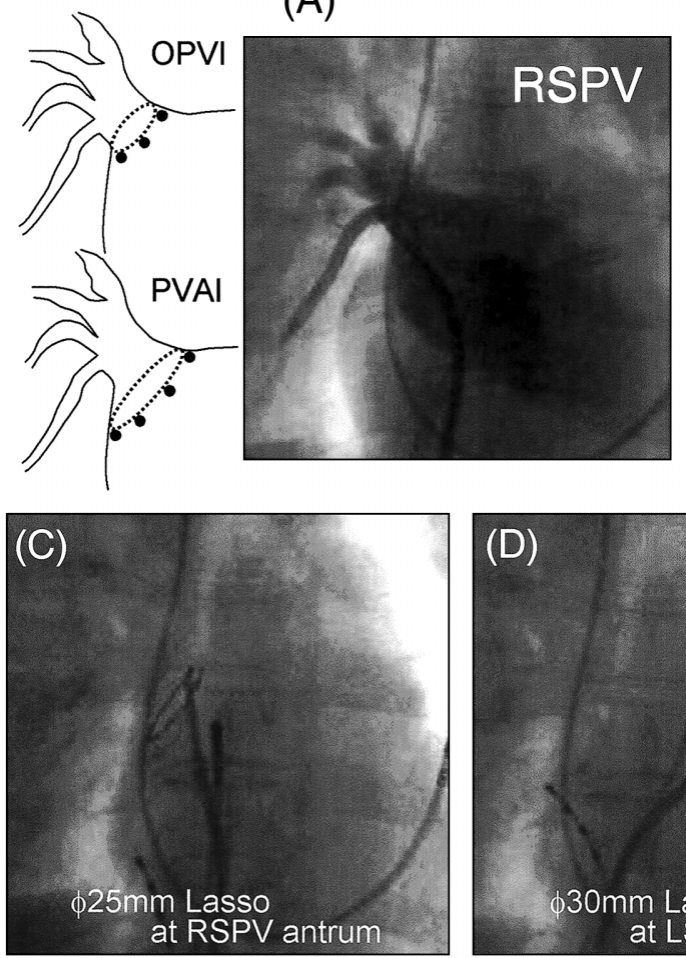

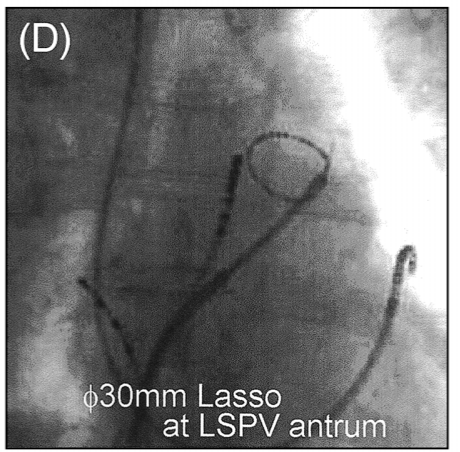

Fig 1. (A, B) Angiographic image of both superior pulmonary veins (PVs). The afjacent schema show the target sites of both ostial PV isolation (OPVI) and PV antral isolation (PVAI). The dotted circle is the position of the Lasso catheters, and the black dots rare the radiofrequency (RF) application sites. (C, D) In this case, Lasso catheters with a diameter of $30 \mathrm{~mm}$ and $25 \mathrm{~mm}$ were chosen for the left superior and right superior PV (LSPV and RSPV), respectively, to perform PVAI, according to the diameter of the PV antrum measured on the angiographic image. (E) Three-dimensional re-constructed view of the intracardiac echocardiology image during PV antrum mapping. White arrowheads indicate the ostium of the right superior PV, black arrows indicate the ring configuration of the 30-mm Lasso catheter positioned at the antrum of this vein.

tions at Jikei University Hospital. No patient dropped out during the observation period. Informed consent was given by each patient before the procedure, according to the protocol approved by the hospital's Human Research Committee.

\section{Mapping Techniques}

Mapping and ablation was performed as described previously? Briefly, the procedures were performed 7 days after the withdrawal of antiarrhythmic drugs (no patient took amiodarone). The LA and PVs were explored through either a patent foramen ovale (19 patients) or transseptal catheterization with 2-3 catheters: 1-2 for circumferential PV mapping, and a quadripolar mapping/ablation catheter. The direct visualization of all 4 PVs was performed using selective venography through a long sheath $(8 \mathrm{~F}$ (SR-0, DAIG company, USA) for superior PVs; 8F percutaneous transseptal introducer sheath (Medtronic, USA) for inferior PVs) after manual injection of contrast medium. Heparin was titrated to maintain a partial thromboplastin time of $60-90 \mathrm{~s}$ (control =30 s).

The PV ostium and antrum were identified on the angiographic image (Figs 1A,B). As has been previously described, the PV ostium was angiographically defined as the region where the outline from the LA to PV curves with a maximum angle,$^{15}$ whereas the PV antrum was defined as the intervening area between the venous tube and the widely opened LA, which was identified angiographically as the gradually widening region around the PV ostium. PV mapping was performed using a steerable circular catheter with a diameter of either 15, 20, 25 or $30 \mathrm{~mm}$ equipped with 20 1 -mm electrodes in a loop made of shape-retaining material orthogonal to the shaft (Lasso, Biosense Webster, Diamond Bar, CA, USA). In the Group 1 patients, we mapped and ablated the PV ostium according to the conventional method, 1,7 using a standard Lasso catheter ( 15 or $20 \mathrm{~mm}$ in diameter), whereas larger sized Lasso catheters (diameter of 25 or $30 \mathrm{~mm}$ ) were used for mapping the PV antrum in the Group 2 patients. In practise, we chose the Lasso catheter based on the roughly measured diameter of the PV ostium or antrum according to the angiographic image (Figs 1A-D). In order to improve the contact between the large-sized Lasso catheter and the surrounding wall of the PV antrum (outside of the PV ostium) for better mapping, we used a catheter holder stabilizer (custom-made) which helps to maintain the position of the ring catheter against the LA wall and thus minimize its movement. The close contact of the ring configuration of the Lasso catheter with the PV antral wall was confirmed in 3 patients of Group 2 by intracardiac echocardiography (USE-1200, Tom Tec Imaging Systems, Germany) (Fig 1E). In both groups, the local potentials (PV muscle potentials in OPVI or PV antral potentials in PVAI) were recorded in the bipolar mode from 10 bipoles $(1-2,3-4, \ldots$, up to $19-20$ with the initial electrode as the anode and the next electrode as the cathode) through bandpass filters of $30-500 \mathrm{~Hz}$ and an amplification of $1-2 \mathrm{~cm} / \mathrm{mV}$ on a polygraph (EPMed Systems, Inc, West Berlin, NJ, USA). 
(A)

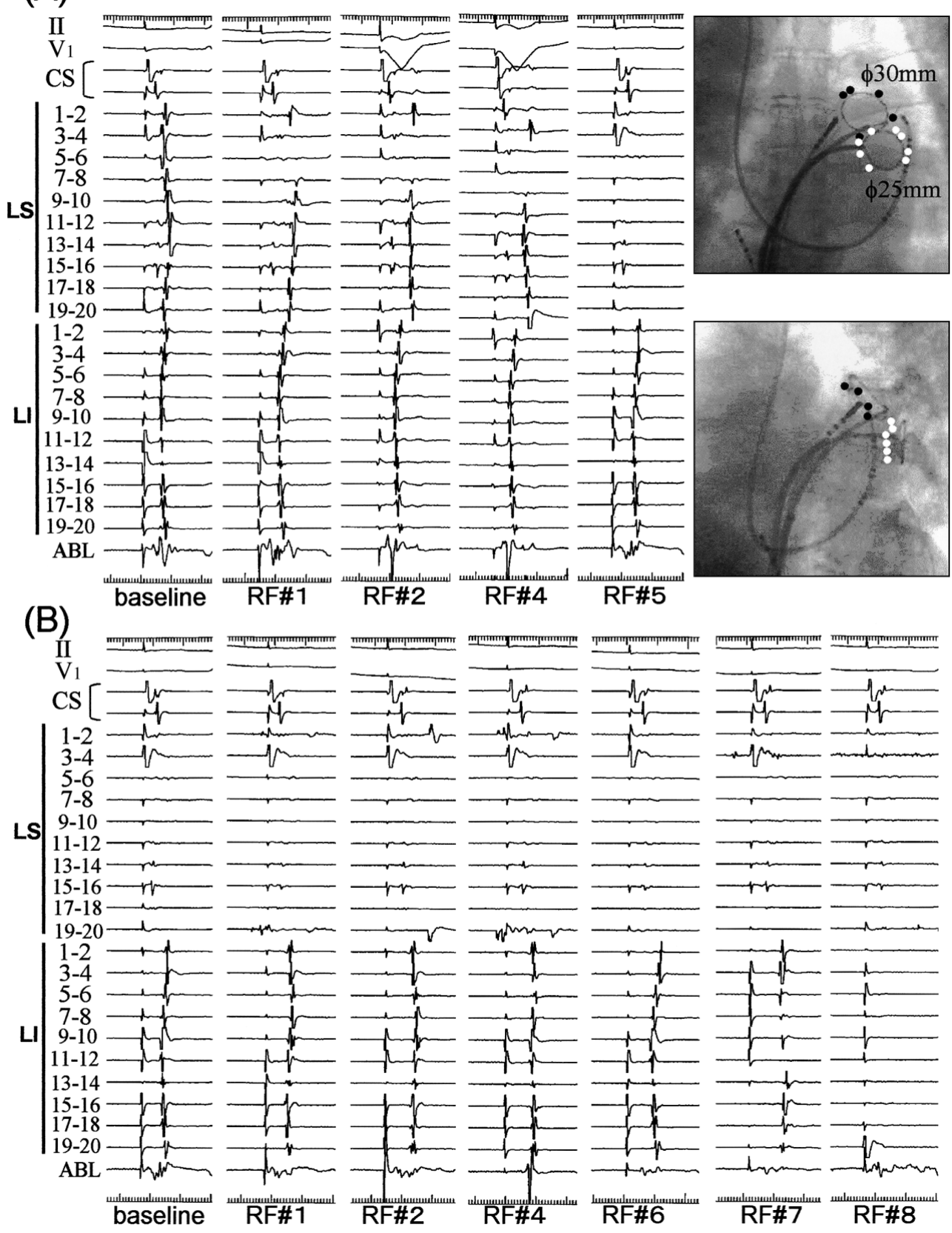

Fig 2. Intracardiac recordings and catheter positions during segmental PVAI under sinus rhythm. Two Lasso catheters with a diameter of $30 \mathrm{~mm}$ and $25 \mathrm{~mm}$ were positioned at the antrum of the LSPV and left inferior PV (LIPV), respectively (images). (A) At baseline, \#3-4 and \#5-6 of the bipolar recordings in the LSPV and \#7-8 and \#9-10 of the LIPV show both the earliest activation and the polarity reversal. Segmental RF application adjacent to \#3-4 and \#5-6 where the local potential recorded by the ablation catheter showed fractionated potential, eliminated the PV potentials distal to the ablation site. Subsequent segmental RF applications targeting the new earliest activation sites at the antrum of the LSPV gradually altered the local activation sequence and finally $5^{\text {th }} \mathrm{RF}$ application succeeded in disconnecting this vein. (B) Similarly in the LIPV, the activation sequence gradually changed and all the potentials disappeared by the $8^{\text {th }}$ segmental RF application at the antrum. Other abbreviations see in Fig 1.

\section{Ablation Procedure}

In each case, all $4 \mathrm{PVs}$ were electrically disconnected from the LA, except if the PV had a diameter less than $12 \mathrm{~mm}$ and no arrhythmogenicity. In cases with SR, the segments of the PV perimeter demonstrating the earliest activation with the electrogram polarity reversal were preferentially targeted? In patients who underwent PVI during ongoing $\mathrm{AF}$, the segments demonstrating either fraction- ated electrograms during disorganized local potential activation, or electrogram polarity reversal, or the earliest activation during the transient or sustained organization of the local potential activation, were preferentially targeted (Figs 3A,B)?,10 RF energy was delivered at the distal electrode (8-mm tip) of the thermocouple-equipped ablation catheter (Fantasista, Japan Lifeline, Tokyo, Japan, or Blazer II, Boston Scientific, CA, USA) with a target temperature 


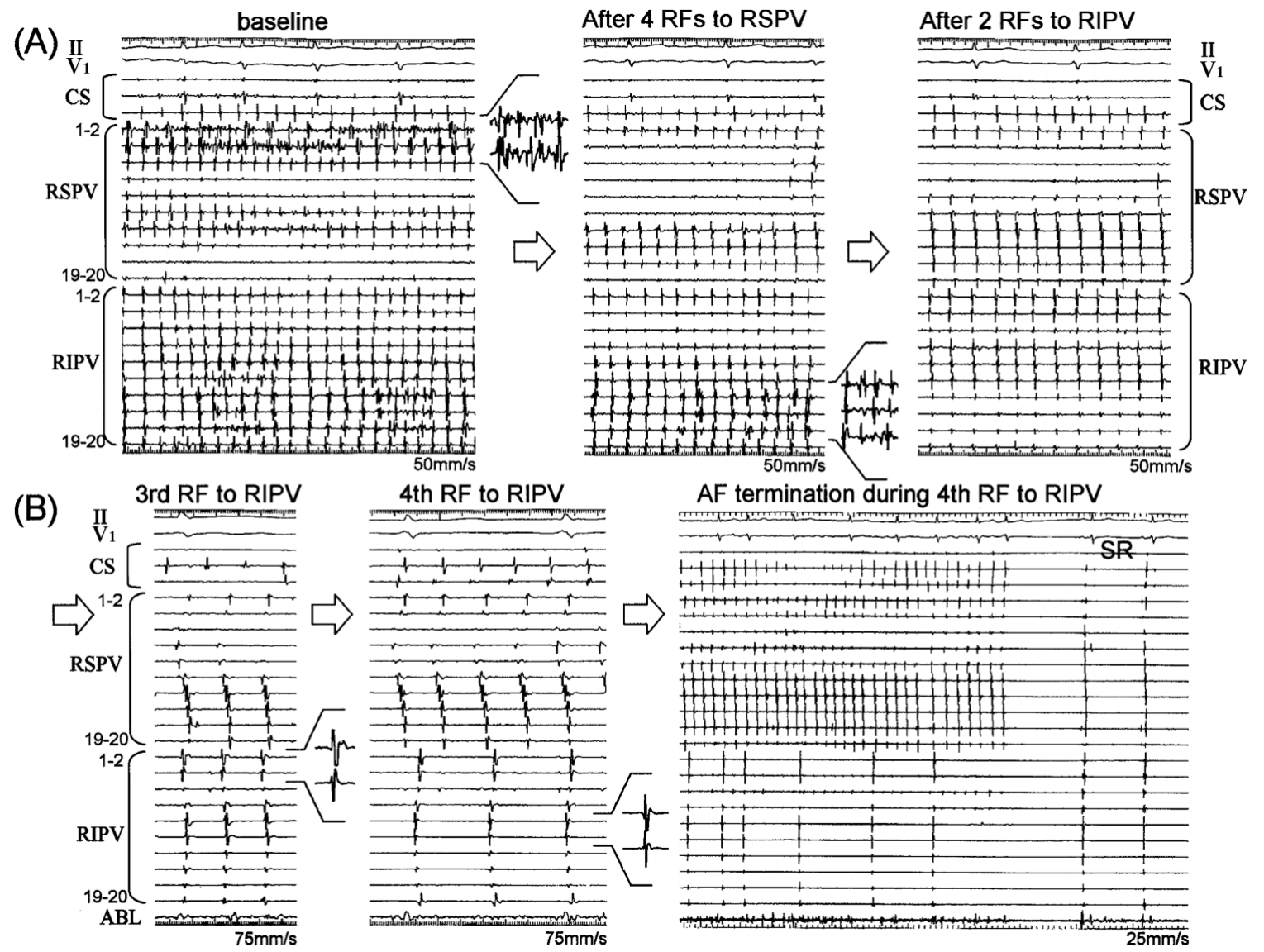

Fig 3. Typical example of PVAI during ongoing atrial fibrillation (AF). In each panel, the targeted potentials on the Lasso catheter are magnified and shown in the inset. (A) At baseline, both the right superior and inferior PV show localized fragmented activity around the antrum. RF applications adjacent to these fractionated potentials gradually organize the local potentials on the Lasso catheter. Similarly, RF applications at the right inferior PV (RIPV) antrum showing fractionated potentials successfully eliminated the disorganized activity around this vein. (B) Successive recordings from Panel A. Once the PV antral potentials became organized by RF applications during ongoing AF, the regions showing the earliest activation with polarity reversal were targeted. As shown in the left panel, the first 2 recordings (\#1-2 and \#3-4) in the RIPV demonstrate the earliest activation with polarity reversal (inset). A single RF application to this region slowed down the activation frequencies in this vein, as well as alterating the activation sequence (\#9-10 and \#11-12 became the earliest). Another RF application adjacent to electrodes \#9-12 further slowed down the activation frequency of this vein and then terminated the AF (before the complete disconnection of this vein). Other abbreviations see in Figs 1,2.

of $50^{\circ} \mathrm{C}$ and a power limit of $30-35 \mathrm{~W}$ for $30-60$ s each site. In order to avoid char formation on the circular mapping catheter, we ablated not on the mapping catheter directly, but proximally to the electrodes of the Lasso catheter.

The endpoint of ablation in both groups was the establishment of a bidirectional conduction block between the LA and PV. After elimination of PV muscle conduction distal to the ablation site(s), indicated either by the abolition or dissociation of distal PVPs, the absence of conduction from the PV to LA was also confirmed by pacing inside the PV by the mapping catheter or the Lasso catheter during SR!1,12 After electrical disconnection of the targeted PVs, provocative maneuvers (isoproterenol and burst pacing) were performed to reveal other remaining foci in the ostium proximal to the ablation sites or in other atrial tissues. Additional RF ablation was performed, targeting these remaining foci if necessary.

The position of the esophagus behind the LA was monitored throughout the ablation procedure in approximately half of Group 2 patients (64 patients), in order to avoid esophageal damage from the RF energy applications. As described previously, direct esophagraphy was performed by infusing water-soluble contrast medium (approx. $4-8 \mathrm{ml}$ amidotrizoic acid, Schering AG, Germany) through a nasogastric tube under deep sedation and retaining it in the esophagus throughout the procedure to reveal its silhouette. Real-time monitoring of the esophageal location enabled ablation to be carried out safely.

No continuous ablation lines were produced in this patient population, except for 2 atrial tachycardias in 2 patients in Group 2, which appeared after the PVAI procedure (described in Results).

\section{Patient Follow-up After Ablation}

All patients remained in hospital for at least 4 days after the procedure, with continuous monitoring of the ECG. After being discharged, the patients underwent careful observation ( 2 weeks after discharge, then monthly) at the cardiology clinic, without taking any antiarrhythmic agents. The outcome of PVI was evaluated by patient symptoms, ECG at periodical follow-up, and also by periodically performing 24-h ambulatory monitoring (at 1 day, and 1, 3, 
6, 9, and 12 months after the procedure). If the patients complained of any symptoms suggestive of tachycardia, a cardiac event recorder (CG-6106, Card Guard Scientific Survival, Rehovot, Israel) was used for 5 successive days to define the cause of symptoms. In the case of early AF recurrence during admission, either a drip-infusion of antiarrhythmic drugs was administered or electrical cardioversion was performed. In general, the patients were discharged on warfarin anticoagulation (continued for 6 to 12 months after the procedure), but without any antiarrhythmic therapy.

The outcome of the procedure was generally evaluated 3 months after the procedure. AF recurrence within the first month after ablation was not counted in the analysis and those who did not have any evidence of tachycardia after more than 1 month of follow-up were considered to be "successful". In cases of AF recurrence with severe symptoms, antiarrhythmic drugs, which had been ineffective before the procedure, were used either temporarily or continuously. The appearance of AF more than 3 months into the observation period, with or without antiarrhythmic drugs, was considered as "failure of the procedure" and a repeat ablation $(\mathrm{ABL})\left(2^{\text {nd }}\right.$ or $3^{\text {rd }}$ procedure) was recommended.

\section{Statistical Analysis}

All values are expressed as the mean \pm SD. A statistical analysis was performed using Student's t-test (paired or unpaired) and proportions were compared with the chisquare test. Kaplan-Meier analysis with a log-rank test was used to determine the probability of freedom from recurrent AF. Differences with a p-value $<0.05$ were considered to be statistically significant.

\section{Results}

Fig 2 is representative intracardiac recordings and catheter positions during segmental PVAI in a patient from Group 2. Two Lasso catheters with diameters of $30 \mathrm{~mm}$ and $25 \mathrm{~mm}$ were positioned at the antrum of the left superior and left inferior PV (LSPV, LIPV), respectively, without any space between the 2 catheters. Segmental RF applications at the antrum of the LSPV gradually altered the activation sequence of the PV antral potential and finally the $5^{\text {th }} \mathrm{RF}$ application succeeded in disconnecting this vein (Fig 2A). In the LIPV, all potentials were gradually eliminated with 8 RF applications (Fig 2B). It is notable that most of the local potentials at the successful ablation sites in both veins showed fragmented electrograms between the atrial and PV potentials, which would reflect delayed conduction along the LA-PV electrical breakthrough. In the LIPV, an additional RF application at the anterior wall was necessary to eliminate the remaining PV-LA conduction and to attain a bidirectional conduction block. These residual PV-LA conductions in the absence of LA-PV conduction were observed in $27.4 \%(79 / 274)$ and $25.0 \%$ (114/458) of the targeted PVs in Group 1 and 2, respectively ( $\mathrm{p}=\mathrm{NS})$.

Fig 3 is a typical example of PVAI in a patient from Group 2 who had persistent AF. At baseline, both superior and inferior PVs showed localized fragmented activity around their antrums. RF applications targeting these regions eliminated the fragmentation, thus resulting in organization of the PV antral potentials (Fig 3A). Once the local potentials became organized, we could easily identify the residual breakthrough point from the LA to the PV antrum, even during ongoing AF. As shown in Fig 3B, we next
Table 2 Comparison of Ablation Results in the 2 Groups

\begin{tabular}{|c|c|c|c|}
\hline & $\begin{array}{c}\text { Group } 1 \\
(n=70)\end{array}$ & $\begin{array}{l}\text { Group } 2 \\
(n=117)\end{array}$ & $p$ value \\
\hline$\%$ of isolated $P V$ & $99 \%$ & $99 \%$ & NS \\
\hline RF numbers/4-PVI & $24 \pm 7$ & $38 \pm 11$ & $<0.01$ \\
\hline$R F$ duration (min) & $22 \pm 7$ & $36 \pm 9$ & $<0.01$ \\
\hline Procedure time $(h)$ & $4.8 \pm 1.2$ & $5.2 \pm 1.1$ & $<0.05$ \\
\hline Fluoroscopic time $(h)$ & $49 \pm 18$ & $56 \pm 21$ & $<0.05$ \\
\hline \multicolumn{4}{|l|}{ No. of ablated sectors } \\
\hline Superior PVs & $4.4 \pm 1.5$ & $6.1 \pm 1.1$ & $<0.01$ \\
\hline Inferior PVs & $3.5 \pm 1.3$ & $4.3 \pm 1.4$ & $<0.05$ \\
\hline Drug use (temporary) & $7(10 \%)$ & $8(7 \%)$ & $N S$ \\
\hline Drug use (continuous) & $11(16 \%)$ & $14(12 \%)$ & NS \\
\hline No. of ABL procedures & $1.4 \pm 0.4$ & $1.4 \pm 0.5$ & $N S$ \\
\hline Non- $P V$ foci in repeat $A B L$ & $22(31 \%)$ & $16(14 \%)$ & $<0.01$ \\
\hline Around PV ostium & $12(17 \%)$ & $4(3 \%)$ & $<0.05$ \\
\hline SVC & $6(8 \%)$ & $12(10 \%)$ & $N S$ \\
\hline \multicolumn{4}{|l|}{ Complications } \\
\hline PV stenosis & 3 & 0 & $<0.05$ \\
\hline LA flutter & 1 & 4 & $N S$ \\
\hline
\end{tabular}

Group 1 represents patients who underwent ostial pulmonary vein isolation; Group 2 represents patients who underwent pulmonary vein antrum isolation.

$P V$, pulmonary vein; $R F$, radiofrequency; $A B L$, ablation; SVC, superior vena cava. Other abbreviation see in Table 1.

targeted the region with the earliest activation and the electrical polarity reversal (inset of Fig 3B) at electrode \#1-2, which slowed down the activation frequencies in this vein, as well as the alteration of the activation sequence. Another RF application (4th $A B L$ ) adjacent to electrodes \#9-12 further slowed down the activation frequency of this vein and then terminated the AF (before the complete disconnection of this vein). In total, RF applications at the segments of fragmented activities around the PV antrum during AF were attempted in 56 cases from Group 2, which resulted in organization of local activity and termination of the AF in 22 cases (39\%). RF application around the PV ostium targeting the fragmented activities during AF in 28 cases of Group 1 resulted in AF termination in only 6 cases $(21 \%$, a significantly lower rate than that observed in Group 2, $\mathrm{p}<0.05)$.

The results of ablation are shown in Table 2. A total of 736 PVs were ablated among 187 patients in both groups of this study, including 187 LSPV, 187 RSPV, 177 LIPV, and 185 RIPVs. Nine left PVs with a common trunk were counted as LSPV (2 in Group 1, 7 in Group 2). Although there was no significant difference between the 2 groups regarding the success rate of isolating target PVs (99\% in each group), PVAI required a significantly longer RF application time during a longer procedure time compared with OPVI (Table 2). The extent of the regions where RF energy was effectively applied was also compared between the 2 groups. When we divided the PV-ostial or PV-antral perimeter into 8 segments, $4.4 \pm 1.5$ sectors and $6.1 \pm 1.1$ sectors of the superior PVs had to be ablated in Groups 1 and 2, respectively $(\mathrm{p}<0.01)$. Similarly in the inferior PVs, significantly more sectors needed to be ablated with PVAI than with OPVI (3.5 \pm 1.1 vs $4.3 \pm 1.4$ sectors in Groups 1 and 2, respectively, $\mathrm{p}<0.05)$. All the circumferential RF energy applications were required in $8 \%$ and $15 \%$ of PVs in Group 1 and 2 patients, respectively $(\mathrm{p}<0.05)$.

Fig 4 demonstrates the AF-free survival curve after the initial and final PVI in both groups. In the Group 1 patients, the AF-free ratio after the first procedure was $58 \%$ and $32 \%$ in paroxysmal and persistent AF patients, respectively 
(A) Result of the initial procedure

\section{i) Group 1}

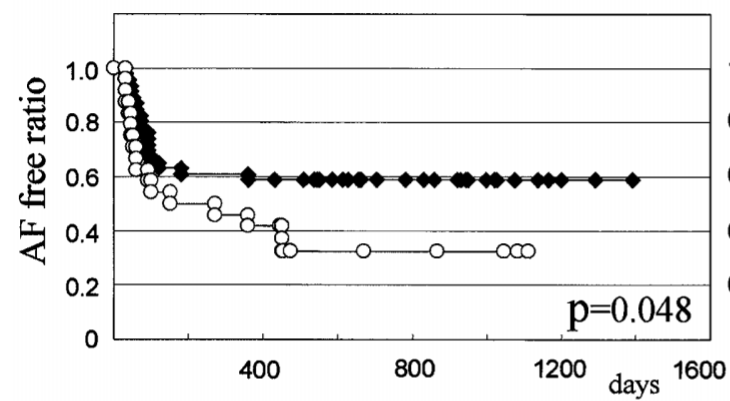

ii) Group 2

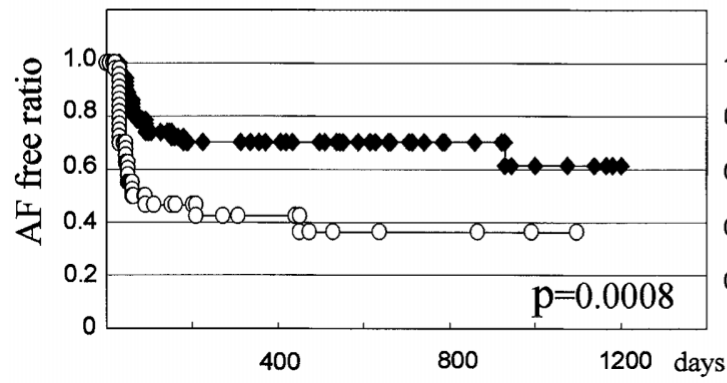

(B) Result of the final procedure

i) Group 1

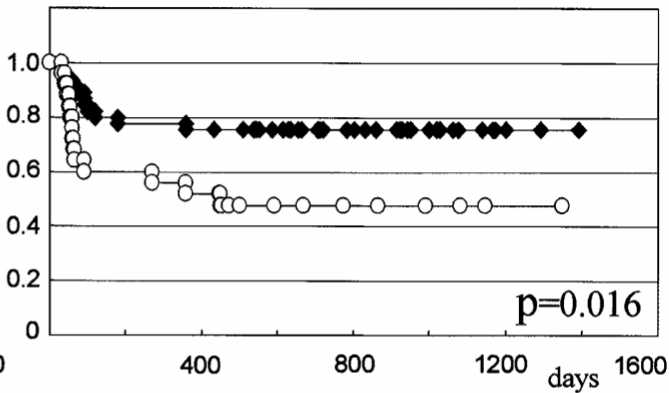

ii) Group 2

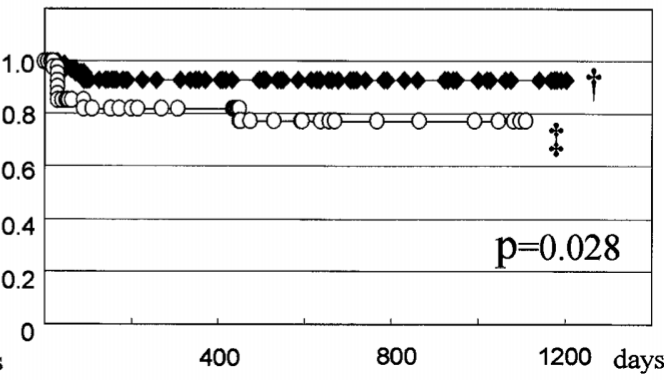

Fig 4. Kaplan-Meier curve of the AF-free survival following either the initial (Panel A) or final PV isolation (Panel B) in both groups. Panel (A) In Group 1 patients, the AF-free ratio was $58.7 \%$ and $32.4 \%$ for paroxysmal and persistent AF, respectively, following the initial procedure ( $\mathrm{p}=0.052$ ), whereas $61.4 \%$ and $36.2 \%$ of the respective Group 2 patients were free from AF following the initial procedure ( $\mathrm{p}=0.0008$ ). There was no significant difference between the 2 groups in the AF-free ratio. Panel (B) The AF free ratio following the final procedure in each group (mean procedure number: 1.5). In the Group 1 patients, the AF-free ratio was $75.6 \%$ and $47.7 \%$ for paroxysmal and persistent, respectively $(\mathrm{p}=0.016)$, compared with $92.7 \%$ and $77.5 \%$ of the respective patients in Group 2 ( $\mathrm{p}=0.028$ ). Significant difference between the 2 groups was observed in the AF-free ratio after the final procedure regardless of the type of AF (Group 1 vs 2: 75.6\% vs

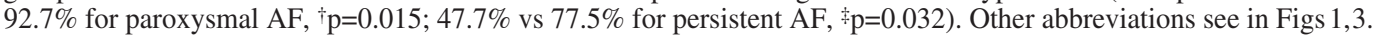

$(\mathrm{p}=0.048)$, whereas $62 \%$ and $36 \%$ of Group 2 patients, respectively, were free from $\mathrm{AF}$ after the first procedure ( $\mathrm{p}=0.0008$, Fig $4 \mathrm{~A}$ i\&ii). After the first procedure, no significant difference was observed in the AF-free ratio between the 2 groups. Repeat ablation sessions were performed in 39\% (27/70) and 37\% (43/117) of patients with a mean procedure number of $1.4 \pm 0.4$ and $1.4 \pm 0.5$ times in Group 1 and 2 , respectively ( $\mathrm{p}=\mathrm{NS})$. A third procedure was performed in 2 and 5 patients in Group 1 and 2, respectively. Regarding the final outcome, in Group $176 \%$ and $48 \%$ of the patients with paroxysmal and persistent AF became free from $\mathrm{AF}(\mathrm{p}=0.016)$ compared with $93 \%$ and $78 \%$ of the respective patients in Group $2(\mathrm{p}=0.028)$. The AF-free ratio after the final procedure was significantly higher in Group 2 than in Group 1, regardless of the type of AF $(93 \%$ vs $76 \%$ for paroxysmal $A F, p=0.015 ; 78 \%$ vs $48 \%$ for persistent $\mathrm{AF}, \mathrm{p}=0.032$ ).

It is noteworthy that there was a significant difference between the 2 groups in the cause of AF recurrence after the first procedure (Table2). Ectopic firing from residual tissue around the PV ostium occurred more frequently in Group 1 than in Group 2 during the second session (17\% and 3\%, respectively, of patients in Groups 1 and 2 who underwent the second session $(\mathrm{p}<0.05))$. However, recurrent conduction of previously isolated PV was similarly found in both groups $(100 \%$ of patients who underwent the $2^{\text {nd }}$ session in both Groups 1 and 2). The average number of re-conducted PVs was also similar in both groups $(2.4 \pm 1.1$ vs $2.3 \pm 1.4 \mathrm{PVs}$ in the $2^{\text {nd }}$ procedure, $2.0 \pm 0.0$ vs $2.0 \pm 0.7$ $\mathrm{PVs}$ in the $3^{\text {rd }}$ procedure, in Groups 1 and 2, respectively, $\mathrm{p}=\mathrm{NS})$. There were no life-threatening complications in the study population, except 3 cases of PV stenosis (single vein in each patient) in Group 1. Left atrial flutter, which newly appeared after the procedure, was observed in 1 and 4 patients in Group 1 and 2, respectively ( $\mathrm{p}=\mathrm{NS}$ ). All instances of LA flutter were successfully eliminated during repeat ablation sessions, 3 cases of which were related to recurrent PV conduction and a peri-mitral reentry was documented in 2 patients, both of which were successfully eliminated by left isthmus ablation ${ }^{14}$ No continuous ablation lines were produced in this patient population, except for the above 2 cases of peri-mitral reentrant tachycardia in Group 2.

\section{Discussion}

Segmental PVI using a circular mapping catheter was originally developed by Haissaguerre et $\mathrm{al}^{1}$ in order to disconnect arrhythmogenic PVs from the LA at their ostia. Because the diameter of PV at its ostium has been estimated to be approximately $15-20 \mathrm{~mm}, 15$ Lasso catheters with equivalent diameters are generally used for OPVI!,2 Although the efficiency of OPVI in suppressing the occurrence of paroxysmal AF has been well established, several problems relating to the technique have also been identified, such as residual ectopic foci arising from arrhythmogenic tissue remaining around the PV ostium ${ }^{16}$ or because of PV 
ostial narrowing? On the other hand, wide-area circumferential ablation around both ipsilateral superior and inferior PVs, which has been developed and used widely, also results in de novo complications from linear RF application in the LA, such as atrioesophageal fistula ${ }^{17}$ and LA flutter ${ }^{18}$ In the present study, we aimed to isolate each of the $4 \mathrm{PVs}$ at its antrum under the guidance of activation-mapping using large-sized Lasso catheters and we compared the results of this approach with those for the conventional PV ostial isolation. We showed that (i) segmental PVAI is feasible using the large-sized Lasso catheter, although a significantly wider region is required to ablate compared with OPVI, and (ii) segmental PVAI is safer and more effective than OPVI, especially in cases of persistent AF. As far as we are concerned, there has not been a previous report describing the feasibility, efficiency and safety of segmental PVAI using large-sized Lasso catheters.

Haïssaguerre et al reported that nearly half of the PV perimeter must be ablated for electrical isolation of a PV at its ostium, whereas Marrouche et al reported that almost the entire PV perimeter must be targeted for proximal isolation of a PV 19 One of the major new findings of the present study is that proximal isolation of a PV at its antrum can be achieved in an EP-guided segmental manner, thus using the same strategy as for the OPVI, but without making linear ablation lesions. Large-sized Lasso catheters with a diameter of either 25 or $30 \mathrm{~mm}$ can fit well at the antrum of each PV and reveal those segments of the PV perimeter that demonstrate the earliest activation or electrical polarity reversal, which suggest the location of LA-PV breakthroughs. Although isolation of a PV at its antrum required a significantly longer period of RF application and greater density of lesions than conventional OPVI, it had a higher AF-free survival ratio and a lower incidence of serious complications such as PV stenosis.

Debate remains over the anatomical substrate of the electrical breakthrough between the LA and PVs. There have been only a few anatomical reports of the complex architecture of the prolonged myocardial sleeves extending into the PVs ${ }^{20-23}$ Ho et al ${ }^{20}$ described that, in addition to the circular or spirally oriented bundles of fibers, there are adjacent longitudinal or obliquely oriented fibers in an intricate and mesh-like arrangement. Such longitudinal fascicles have been reported to be broader proximally and to then branch or become thinner distally, which may thus explain the greater number of RF applications required in Group 2 than in Group 1.

Nademanee et $\mathrm{al}^{9}$ recently reported that areas with complex fractionated electrograms represent a defined electrophysiologic substrate of AF that is an ideal target for ablation. They found that the region around each PV was on of the areas where fractionated electrograms are frequently observed. Haïssaguerre et al ${ }^{24}$ also demonstrated that ablation of regions displaying rapid or heterogenous activation could often prolong the AF cycle length and terminate AF. In the present study, we preferentially targeted segments of the PV antrum with fractionated electrograms when we ablated during $\mathrm{AF}$, which frequently organized these electrograms or terminated the ongoing AF (Fig 3). Regions with fragmented activity around the ipsilateral superior and inferior PV antrum could be identified simultaneously using the large-sized Lasso catheter, thus suggesting its usefulness for not only for the isolation of PV at its antrum but also for identifying and eliminating AF substrate around the PV antrum.
PVAI with a roving Lasso technique has been recently developed by Verma et al, who mapped and ablated each $\mathrm{PV}$ at its antrum under the guidance of a standard 20-mm Lasso catheter and intracardiac echocardiography. Because the area of the PV antrum is larger than the 20-mm Lasso, they sequentially repositioned the Lasso along each segment of the antral circumference, resulting in an excellent outcome for cure of AF. Although our method of PVAI has some similarities to their method, it is unique because the entire PV antral circumference can be mapped at once with the larger-sized Lasso catheter. In both Verma et al's report and the present study, there were no complications involving the esophagus, even though the ablation target regions in PVAI are sometimes very close to it. Thus, it might be beneficial to segmentally apply RF energy with/without titrating it under microbubble monitoring6 Real-time monitoring of the esophagus with either intracardiac echocardiography ${ }^{25}$ or continuous esophagraphy ${ }^{8}$ would further improve the safety of PVAI.

There may be discussion about the evaluation of the ablation outcome after multiple procedures. Because it is now well recognized that resumption of conduction in previously isolated PVs can occur in most cases with AF recurrence and that re-isolation of these PVs leads to marked improvement in ablation outcome ${ }^{26-28}$ repeat procedures might be regarded as a part of a stepwise approach to more complete isolation of targeted PVs.

\section{Study Limitations}

This study was not randomized with 2 groups of patients treated during different periods. Although the basic patient characteristics were similar in both groups, operator learning experience might contribute in some degree to the better outcome of Group 2 (more operator experience) compared with Group 1. Because the recurrences were quantified according to patient symptoms and serial ECGs and Holter monitoring, our criterion of $\mathrm{AF}$ recurrence may thus have underestimated the true recurrence rate by not identifying asymptomatic AF recurrence.

\section{Conclusions}

Segmental antral PVI with electrophysiological guidance is feasible using the large-sized $(25-30 \mathrm{~mm})$ Lasso catheters. Antral PVI was found to be more effective and safer than the conventional ostial PVI for the treatment of paroxysmal and persistent AF.

\section{References}

1. Haïssaguerre M, Shah DC, Jaïs P, Hocini M, Yamane T, Deisenhofer I, et al. Electrophysiological breakthroughs from the left atrium to the pulmonary veins. Circulation 2000; 102: 2463-2465.

2. Oral H, Knight BP, Tada H, Özaydin M, Chugh A, Hassan S, et al. Pulmonary vein isolation for paroxysmal and persistent atrial fibrillation. Circulation 2002; 105: $1077-1081$.

3. Saad EB, Marrouche NF, Saad CP, Ha E, Bash D, White RD, et al. Pulmonary vein stenosis after catheter ablation of atrial fibrillation: Emergence of a new clinical syndrome. Ann Intern Med 2003; 138: 634-638.

4. Pappone C, Rosanio S, Oreto G, Tocchi M, Gugliotta F, Vicedomini $\mathrm{G}$, et al. Circumferential radiofrequency ablation of pulmonary vein ostia. Circulation 2000; 102: 2619-2628.

5. Ouyang F, Bansch D, Ernst S, Schaumann A, Hachiya H, Chen M, et al. Complete isolation of left atrium surrounding the pulmonary veins: New insights from the double-Lasso technique in paroxysmal atrial fibrillation. Circulation 2004; 110: 2090-2096.

6. Verma A, Marrouche FN, Natale A. Pulmonary vein antrum isolation: Intracardiac echocardiography-guided technique. J Cardiovasc 
Electrophysiol 2004; 15: 1-6.

7. Yamane T, Shah DC, Jaïs P, Hocini M, Deisenhofer I, Choi KJ, et al. Electrogram polarity reversal as an additional indicator of breakthroughs from the left atrium to the pulmonary veins. $J$ Am Coll Cardiol 2002; 39: 1337-1344.

8. Yamane T, Matsuo S, Date T, Mochizuki S. Visualization of the esophagus throughout left atrial catheter ablation for atrial fibrillation. J Cardiovasc Electrophysiol 2006; 17: 105.

9. Nademanee K, McKenzie J, Kosar E, Schwab M, Sunsaneewitayakul B, Vasavakul T, et al. A new approach for catheter ablation of atrial fibrillation: Mapping of the electrophysiologic substrate. J Am Coll Cardiol 2004; 43: 2044-2053.

10. Macle L, Jaïs P, Scavee C, Weerasooriya R, Shah DC, Hocini M, et al. Electrophysiologically guided pulmonary vein isolation during sustained atrial fibrillation. J Cardiovasc Electrophysiol 2003; 14: $255-260$.

11. Gerstenfeld EP, Dixit S, Callans D, Rho R, Rajawat Y, Zado E, et al. Utility of exit block for identifying electrical isolation of the pulmonary veins. J Cardiovasc Electrophysiol 2002; 13: 971-979.

12. Kumagai K, Noguchi H, Ogawa M, Nakashima H, Zhang B, Miura $\mathrm{S}$, et al. New approach to pulmonary vein isolation for atrial fibrillation using a multielectrode basket catheter. Circ J 2006; 70: 88-93.

13. Kato R, Lickfett L, Meininger G, Dickfeld T, Wu R, Juang G, et al. Pulmonary vein anatomy in patients undergoing catheter ablation of atrial fibrillation: Lessons learned by use of magnetic resonance imaging. Circulation 2003; 107: 2004-2010.

14. Jaïs P, Hocini M, Hsu LF, Sanders P, Scavee C, Weerasooriya R, et al. Technique and results of linear ablation at the mitral isthmus. Circulation 2004; 110: 2996-3002.

15. Yamane T, Shah DC, Jaïs P, Hocini M, Peng JT, Deisenhofer I, et al. Dilatation as a marker of pulmonary veins initiating atrial fibrillation. J Interv Card Electrophysiol 2002; 6: 245-249.

16. Shah DC, Haïssaguerre M, Jaïs P, Hocini M. Nonpulmonary vein foci: Do they exist? Pacing Clin Electrophysiol 2003; 26: 16311635.

17. Pappone C, Oral H, Santinelli V, Vicedomini G, Lang CC, Manguso $\mathrm{F}$, et al. Atrio-esophageal fistula as a complication of percutaneous transcatheter ablation of atrial fibrillation. Circulation 2004; 109: $2724-2726$
18. Pappone C, Manguso F, Vicedomini G, Gugliotta F, Santinelli O, Ferro A, et al. Prevention of iatrogenic atrial tachycardia after ablation of atrial fibrillation: A prospective randomized study comparing circumferential pulmonary vein ablation with a modified approach. Circulation 2004; 110: 3036-3042.

19. Marrouche NF, Dresing T, Cole C, Bash D, Saad E, Balaban K, et al. Circular mapping and ablation of the pulmonary vein for treatment of atrial fibrillation: Impact of different catheter technologies. $J \mathrm{Am}$ Coll Cardiol 2002; 40: 464-474.

20. Ho SY, Cabrera JA, Tran VH, Farre J, Anderson RH, SanchezQuintana D. Architecture of the pulmonary veins: Relevance to radiofrequency ablation. Heart 2001; 86: 265-270.

21. Saito T, Waki K, Becker AT. Left atrial myocardial extension onto pulmonary veins in humans: Anatomic observations relevant for atrial arrhythmias. J Cardiovasc Electrophysiol 2000; 11: 888-894.

22. Sanchez JE, Plumb VJ, Epstein AE, Kay N. Evidence for longitudinal and transverse fiber conduction in human pulmonary veins: Relevance for catheter ablation. Circulation 2003; 108: 590-597.

23. Fynn SP, Kalman JM. Pulmonary veins: Anatomy, electrophysiology, tachycardia, and fibrillation. Pacing Clin Electrophysiol 2004; 27: $1547-1559$.

24. Haïssaguerre M, Sanders P, Hocini M, Takahashi Y, Rotter M, Sacher F, et al. Catheter ablation of long-lasting persistent atrial fibrillation: Critical structures for termination. J Cardiovasc Electrophysiol 2005; 16: 1125-1137.

25. Ren JF, Lin D, Marchlinski FE, Callans DJ, Patel V. Esophageal imaging and strategies for avoiding injury during left atrial ablation for atrial fibrillation. Heart Rhythm 2006; 3: 1156-1161.

26. Nanthakumar K, Plumb VJ, Epstein AE, Veenhuyzen GD, Link D, Kay N. Resumption of electrical conduction in previously isolated pulmonary veins: Rationale for a different strategy? Circulation 2004; 109: 1226-1229.

27. Callans DJ, Gerstenfeld EP, Dixit S, Zado E, Vanderhoff M, Ren JF, et al. Efficacy of repeat pulmonary vein isolation procedures in patients with recurrent atrial fibrillation. J Cardiovasc Electrophysiol 2004; 15: 1050-1055.

28. Noguchi H, Kumagai K, Yasuda T, Ogawa M, Tojo H, Saku K. Conduction recovery after pulmonary vein isolation for atrial fibrillation. Circ J 2005; 69: 65-68. 